\title{
UAV Pre-Study for In-Air-Capturing Maneuver
}

\author{
Stefan Krause \\ German Aerospace Center \\ Institute of Flight Systems \\ Department Unmanned Aircraft \\ Lilienthalplatz 7 \\ 38108 Braunschweig, Germany \\ Stefan.Krause@dlr.de
}

\author{
Sebastian Cain \\ German Aerospace Center \\ Institute of Flight Systems \\ Department Unmanned Aircraft \\ Lilienthalplatz 7 \\ 38108 Braunschweig, Germany \\ Sebastian.Cain@dlr.de
}

\begin{abstract}
Implementation of reusable launch vehicle (RLV) missions is a major goal in current aerospace research. One conceptual idea to return a booster stage is the so-called "in-air capturing" (IAC), where a winged stage is captured by an aerial vehicle and towed back to the final destination. This approach has the advantage that a towed winged stage does not need a propulsion system or fuel reserves to arrive to a destination point, compared to alternative approaches as demonstrated by SpaceX.
\end{abstract}

The concept of capturing the RLV in air is based on earlier IAC missions for satellite photo capsules and the probe-and-drogue refueling method. A key difference is that in an air-to-air-refueling procedure a highly dynamic fighter tries to connect to a trailing drogue or rigid boom from a sluggish tanker. Opposite to this procedure, it can be assumed that in IAC case two aerodynamically sluggish aircraft need to be coupled.

The challenge is to build up a formation which enables a connection between a gliding RLV and a dynamic coupling device trailing from a large aircraft. To investigate this IAC approach, the German Aerospace Center (DLR) built a scaled demonstration system with smaller unmanned aerial vehicles (UAV) to research different aspects of IAC flight tests.

Based on the assumption that at an IAC approach would involve two large aerodynamically sluggish systems, a third highly dynamic vehicle should be introduced to enable a safe and reliable connection. Therefore, the trailing system, which is known from the air-to-air refueling, was modified by DLR with aerodynamic control surfaces and an independent flight control system. This enables a dynamic and independent motion of the coupling device relative to the towing aircraft, as well as the RLV.

This paper gives an overview about IAC investigations of DLR, which are validated in experimental flight test demonstrations with scaled unmanned systems. The focus of this work is on building up the formation, from the rough approach with GNSS up to the final approach, where the global, absolute localization is supported by an image based relative position estimation of the coupling device. A major aspect in the formation implementation is the active coupling device (ACD). Therefore, the paper will show the construction of the ACD, its functionality and operation during the formation flight and a validation of its behavior at the flight demonstration.

The first flight test results show that our research is heading in the right direction and further tests are expected to provide comprehensive results for the validation of the IAC concept.
The presented investigations and results base on work from the DLR funded AKIRA project and the subsequent project FALCon funded by the European commission in the H2020 Program. FALCon has the aim to increase the TRL of AKIRA investigations and results.

\section{TABLE OF CONTENTS}

2. MISSION …................................................................2

3. Aerodynamic Controlled Coupling DEVICE ...................................................................3

4. GNSS-BASED FORMATION ................................4

5. IMAGE PROCESSING ............................................6

6. EXPERIMENTAL ANALYSIS ............................7

7. SUMMARY \& OUTLOOK ...............................13

ACKNOWLEDGEMENTS ..........................................13

REFERENCES ......................................................14

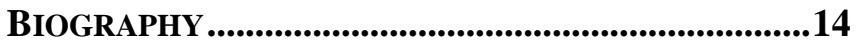

\section{INTRODUCTION}

The main motivation for reusable booster stages or complete spaceships for several missions is reduction of costs [1], [2]. A safe and nondestructive retrieval of the stage from high altitudes to a specified landing position is the key challenge to be solved for reusability. There are several ways to approach such retrieval. One is to use the booster's own propulsion and fuel reserves as demonstrated by SpaceX [1]. Another approach, which is the focus of this paper, is to capture the returning stage with an aircraft which tows it to the landing destination. While this method has yet to be demonstrated, its main advantage would be the reduction of mission fuel and thus an increase of mission payload compared to a retrieval with fuel reserves using the booster's own propulsion [2].

The method of capturing a reusable booster stage followed by a towed flight to a final destination will be referred to as 'In Air Capturing' (IAC). The approach shown in Figure 1 is applied to a winged booster stage comparable to the LFBB [3], a conceptual study developed for the ASTRA program. The booster stage, which will be referred to as the client, has only limited controllability and no propulsion after reentry from 
orbit and will thus have the flight performance of a passive glider with poor flight characteristics and high inertia. The capturing maneuver will be performed at speeds below $\mathrm{Ma}=0.55$ and at flight altitudes between $8,000 \mathrm{~m}$ and 2,000 $\mathrm{m}$, the flight path angle will be at approximately $\gamma=-12^{\circ}$ and the total time available for the maneuver will be approximately 2 minutes [4].

The tow-back airplane will be referred to as the towing aircraft and could be a modified civil or military transport aircraft, which has the necessary excess power for towing the client. However, their high mass and limited maneuverability pose a challenge in the context of the IAC maneuver.

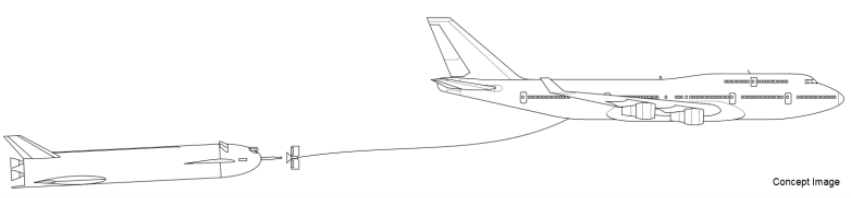

Figure 1: Concept for the IAC method with LFBB and Boeing 747

Regarding the reversible coupling of two aircraft while in flight, an air-to-air refueling maneuver shows similarities to the proposed task. There are several methods for air-to-air refueling which are currently used in practice. One approach is the probe-drogue method [5], which will be used as the basis for IAC. For this procedure, the client, usually an aircraft with high maneuverability which is equipped with a probe, approaches a less agile tanker aircraft that tows the drogue. The more agile client performs the approach while tracking the drogue and compensating for any disturbances. The tanker aircraft maintains steady flight during the maneuver. The same principle can be used for the IAC maneuver but with the additional challenge of establishing contact between a client and a towing aircraft, which both have limited maneuverability and agility. Compensating for disturbances and position deviations by the towing aircraft is less applicable, as control inputs are not transferred to the drogue instantaneously, but are strongly delayed due to the flexible connection between the towing aircraft and the drogue.

In order to allow an in-flight coupling between two aircraft of high mass and limited maneuverability, we propose using an actuated drogue that is not only passively towed, but a controllable device. The objective of this modification is to compensate the limited maneuverability which results from the poor agility of the tanker aircraft and the client. The drogue will therefore be equipped with a control system and aerodynamic control surfaces, which allow active manipulation of its position relative to the client as shown in Figure 2. This controlled drogue is referred to as an 'active coupling device' (ACD).

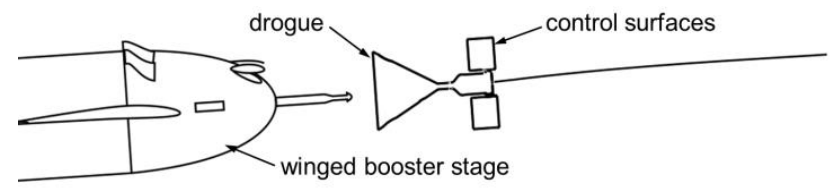

Figure 2: IAC with ACD principle
The approach to equip an air-to-air refueling drogue with aerodynamic control surfaces was also examined in recent studies [6], [7], [8]. The main goal of these studies was to compensate disturbances due to wake vortices or turbulences but not to actively position the drogue and perform the capturing maneuver.

As it is expected to be a high-risk maneuver, it is intended that the returning booster stage as well as the towing aircraft are unmanned and fully automatic. To allow for active positioning of the ACD with respect to the client, it is necessary to estimate the relative position between them. The position estimation can be based on GNSS data or image sensors and is used to calculate the offset, which is compensated by the ACD flight control system. Due to the limited accuracy of GNSS information, the resulting offsets are only suitable for a rough positioning of the vehicles in a first step. A more accurate method, such as image processing, can be used afterwards to control the ACD's position more precisely.

Within the scope of this work, a controller and hardware components were developed for a sub-scale demonstration using small unmanned aircraft. The focus of this effort was to demonstrate an ACD in practice without the cost of a realscale scenario. The underlying concept, technical realization, and validation of the functionality in flight tests are presented in this paper.

\section{MISSION}

Aim of this research is a scaled demonstration of the IAC maneuver, which is the fourth phase of the complete RLV-cycle. The RLV-cycle can be divided into seven phases.

1. Lift-off launcher

2. Main engine cut off of launcher

3. Ballistic trajectory of winged booster stage

4. In-Air-Capturing

5. Towing

6. Separation of towing connection

7. Glide landing of winged stage

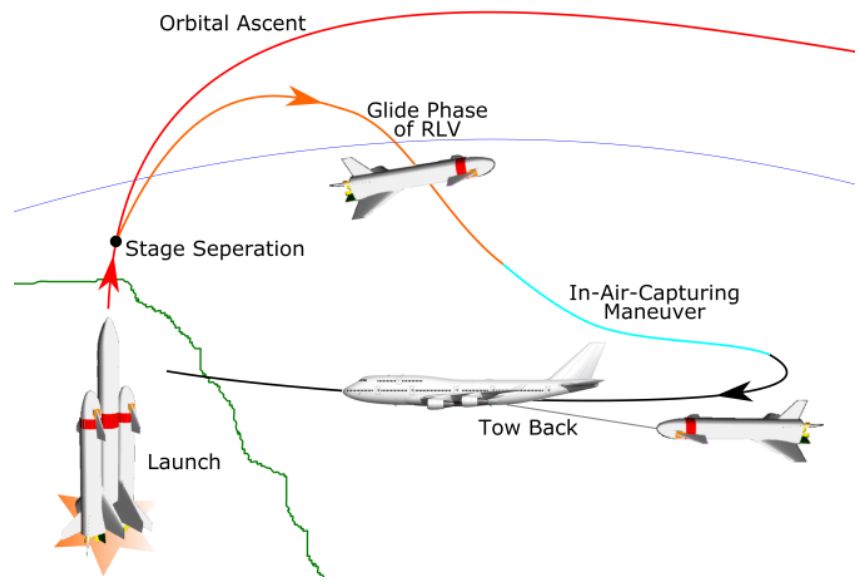

Figure 3: Schematic of the reusable stage's full operational RLV-cycle

As the launcher lifts off the capturing aircraft is waiting 
downrange in the rendezvous area. After main engine cut off the reusable winged booster stage is separated from the rest of the launch vehicle and then follows a ballistic trajectory, soon reaching denser atmospheric layers. At around $20 \mathrm{~km}$ altitude it reaches subsonic velocity and rapidly loses altitude in a gliding flight path. At this point a reusable returning stage usually has to initiate the final landing approach or has to ignite its secondary propulsion system. In contrast, within the IAC method, the reusable stage is awaited by an adequately equipped large capturing aircraft (most likely fully automatic and unmanned), offering sufficient thrust capability to tow a winged launcher stage with limited lift to drag ratio. The entire maneuver is fully subsonic in an altitude range between $8000 \mathrm{~m}$ to $2000 \mathrm{~m}$. After successfully connecting both vehicles with the aid of the ACD, the winged reusable stage is towed by the large carrier aircraft back to the launch site. The stage is then released from its towing aircraft close to the airfield and autonomously glides to the landing runway, similar to a conventional sailplane.

\section{Scaled Mission}

The final approach of the scaled mission differs in several points from the real mission. The scaled maneuver will be implemented in a flight level between approx. $2000 \mathrm{~m}$ and $200 \mathrm{~m}$, with a velocity of around $42 \mathrm{~m} / \mathrm{s}$. The non-propulsive reusable launch vehicle demonstrator (RLVD) will follow a straight path with a $-10^{\circ}$ glide angle.

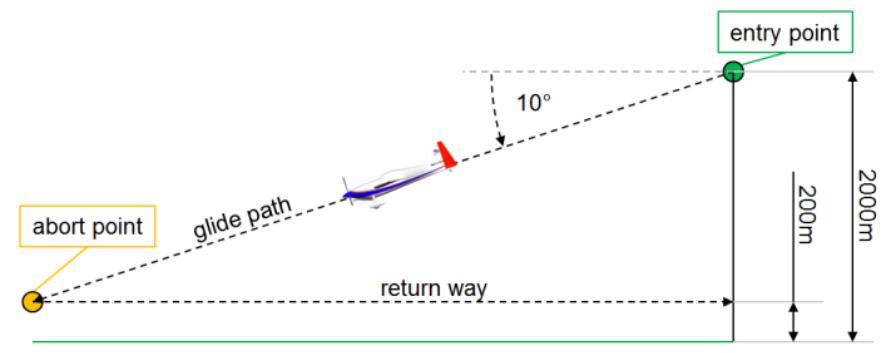

Figure 4: Scenario for the scaled IAC mission

For a coupling approach the Towing Aircraft Demonstrator (TAD) will fly in front of the RLVD in a GNSS-based formation with a horizontal offset of approx. $30 \mathrm{~m}$. The TAD pulls the ACD with an approx. $15 \mathrm{~m}$ rope. This situation represents an outer formation with the aim to build up to a "stable" formation between RLVD and TAD. In the inside of this formation the ACD has the possibility to change its position relative to the outer frame. The motion of the ACD in the inside of the GNSS formation bases on a visual detection of the ACD from the RLVD, with the aim to reduce the deviation between the RLVD and ACD.

To enable an IAC in the final IAC maneuver configuration several sub tasks have be implemented. These steps are an independent development of the ACD, the development of the GNSS-based formation flight, the vision based detection, and movement of the ACD. At the current state of investigations these four sub tasks were developed and validated with simplified maneuvers such as a horizontal race track pattern.

\section{Aerodynamic Controlled Coupling Device}

As mentioned above, the IAC method shares similarities with the air-to-air refueling maneuver, where the drogue is towed by the tanker aircraft and the contact to the probe is established by the client aircraft. For its use within the IAC method, the air-to-air refueling setup must be extended to include a controllable component to compensate the deviations in the relative position of both vehicles. In the ACD case, the drogue is equipped with control surfaces to deflect the drogue with respect to the towing aircraft. The drogue's function is to stabilize the ACD due to its drag in flight and mechanically guide the probe into the coupling mechanism. The aim of the ACD design is to develop a trailing cone with stable flight behavior equipped with a control system and the ability to move orthogonal to the towing aircraft's longitudinal axis $\mathrm{x}_{\mathrm{b}}$. The control surfaces must be able to move the drogue in the range of at least $2 \mathrm{~m}$ in each direction to compensate for offsets resulting from flight performance and GNSS accuracy. Moreover, changes in heading and pitch, $\psi_{\mathrm{ACD}}$ and $\theta_{\mathrm{ACD}}$, should be minimized. For the principal purpose of testing the vertical/lateral movements a simple roll stabilization has to be implemented. The equipment of the device will be stored in the center body of the ACD to have a small moment of inertia around the roll axis. These design aspects apply to a real-scale scenario as well as to the designed scaled device for unmanned testbeds.

For the experimental analysis of the IAC method with smaller unmanned aircraft, an experimental prototype of an ACD was built.

\section{Structure}

Due to the power limitations of the TAD, the weight of the ACD should not exceed $1.0 \mathrm{~kg}$. Also, future modifications should be possible without the need to construct a completely new ACD. Consequently, the prototype was realized as a modular carbon fiber reinforced plastic (CFRP) structure. The main components include:

- center body with avionics, servo motor support and coupling to the towing rope

- adapter from center body to the cone

- cone

- control surfaces including adapter plates to servo motors

The equipment is included in the central body of the unit. To realize the coupling maneuver the control surfaces are oriented in a vertical and horizontal direction. This orientation enables the ACD to directly deflect into orthogonal directions relative to the TAD by using only one pair of surfaces per direction. This statement is based on the assumption that the roll stabilization works without deviations and all axes are perfectly aligned. The control surfaces are mounted directly on the axes of the actuators, so that the installation is very direct and space-saving. This approach requires that the loads on the control surfaces are limited as the bending moments on the servo axes are very high. This limitation was intentionally accepted. 


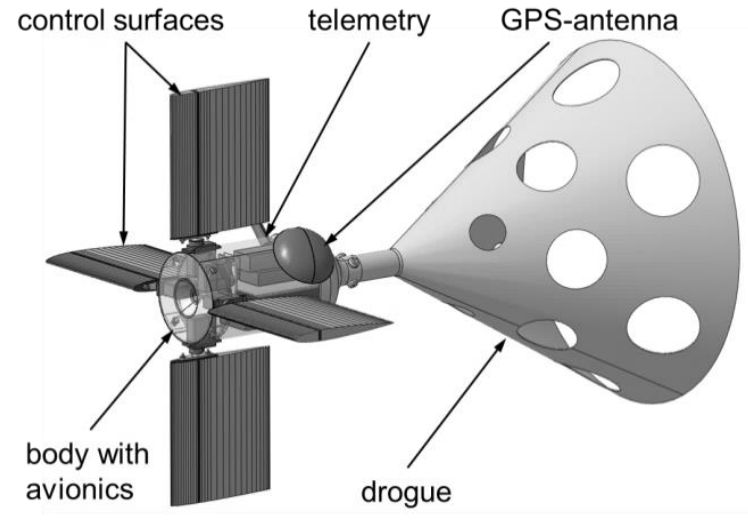

Figure 5: Isometric view of the ACD

The servo motor installations are designed to allow for the quick replacement of damaged parts and have a predetermined breaking point to protect the center body with the avionics. An additional release servo allows for quick decoupling of the ACD from the TAD during landing and failure conditions. In order to minimize turbulence in the cone, it was built with holes, which direct flow energy into the wake space inside of the cone, similar to the design of trailing cones for pressure measurements [9].

Figure 5 shows a 3D model and Table 1 presents some system parameters of the ACD.

Table 1: System parameters of the ACD

\begin{tabular}{|l|l|}
\hline length & $\approx 450 \mathrm{~mm}$ \\
\hline cone diameter & $370 \mathrm{~mm}$ \\
\hline weight & $650 \mathrm{~g}$ \\
\hline control surface span & $120 \mathrm{~mm}$ \\
\hline control surface width & $100 \mathrm{~mm}$ \\
\hline max. control deflection & $+/-45^{\circ}$ \\
\hline
\end{tabular}

\section{Avionics}

The basis for the implementation of control laws for the capturing maneuver is a Pixhawk autopilot [10], an open-source autopilot that provides a very cost-efficient and fully modifiable flight controller. Suitable sensors are available off-theshelf. A u-blox M8N GPS with a compass module, $433 \mathrm{MHz}$ radio telemetry kit, $\mathrm{RC}$ receiver, UBEC voltage regulator and a voltage monitor for the battery are connected to the autopilot. The avionic equipment mounted in the central body of the ACD is shown in Figure 6. Integrated into the autopilot is an inertial measurement unit, from which bank and pitch angle are estimated. For analysis, a data logging tool for sensor and command data is implemented.

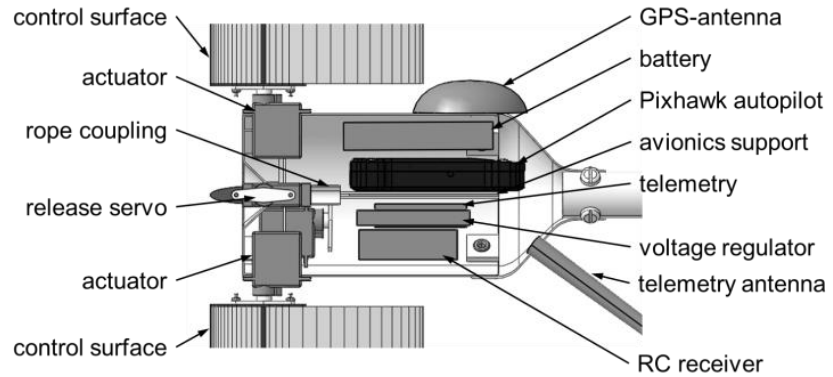

Figure 6: Avionics integrated in the ACD (section view)

The positioning of the ACD as well as the towing aircraft relies on position data from each of the vehicles. As described above, among other things, image sensors can be used to enable and support the final approach. In the case described here image sensors were equipped in the nose of the client to detect and observe the ACD during the final maneuver until the actual docking is completed.

In addition to the link to its ground control station, the ACD must be connected to the client aircraft with a telemetry link. With this connection the relative position data are transmitted as input to the control system of the coupling device.

The control system is realized by adapting the commonly used software of the Pixhawk autopilot. The basic open source software is 'PX4 Firmware', which is based on a 'ChibiOS' real-time operating system that runs sensor drivers and a flight controller. In order to have full control of the actuators, the preinstalled flight management is replaced by a user application generated from a Matlab/ Simulink model with automatic code generation and the 'Pilot Support Package' (PSP) provided by Matlab, including a number of libraries to use Pixhawk interfaces (e.g. sensor data or RC input) within the Simulink environment. Based on this toolchain, a modular, model-based design of the controller with automatic implementation of the generated code into the basic software of the autopilot was realized. For later testing of different control modes, a model structure is constructed, where different, switchable modes are implemented.

The first mode is pure RC control and mixes the commands from the RC channels as asymmetric servo outputs for roll control or symmetric outputs for vertical and lateral movements. A second mode implements a roll stabilization, which ensures that the axes of the control surfaces are always oriented in the right direction. These modes, especially the roll stabilization, are the basic implementations for testing the vertical/lateral displacements without having image processing inputs from a client aircraft. The roll stabilization is realized by applying a classic PID controller to the roll angle. In addition to the commands for the roll stabilization, deflections for vertical/lateral movement are commanded proportionally to the RC inputs.

\section{GNSS-BASED FORMATION}

An idea behind the usage of smaller unmanned systems to validate complex and expensive scenarios is to achieve fast 
and cost-effective results, compared to the use of large and more complex systems. This concept offers the advantages of using existing open source hardware and software with fast development timelines and a great feature base. This decision follows the requirement to reduce the necessary modifications at the open source hardware and software level in order to easily take advantage of future developments within the open source community.

\section{TAD and RLVD autopilots}

Just as the ACD, the TAD and the RLVD are equipped with Pixhawk autopilots. However, the flight control software uses the ArduPlane module from the ArduPilot framework. The MissionPlanner API is used as a ground control station (GCS) to communicate with the flight control software.

ArduPlane contains a lot of functions/modes to control a single fixed-wing aircraft, but a build-up to a formation flight or controlling more than one system at the same time is not directly supported.

Within the scope of this paper, formation is defined as a geometric arrangement of two or more vehicles. The maneuver or the coordination of the formation is agreed before the implementation is initialized. Only one participant has the lead.

\section{Communication Structure}

A requirement to build up a formation is the communication between the participants of the formation. This includes an aircraft-to-aircraft communication module and the basic open source flight controller to manage the flight behavior of each participant. As described initially, the modification of the basic controller should be limited to a minimum to prevent a separation from the open source community development to easily merge future developments.

The necessary scope of modifications to the open source controller can vary depending on the kind of implementation. Therefore, several solutions to integrate the necessary communication structures in the ArduPlane controller were validated.

Participants of the formation are described as one master node and one slave node. The implementation of the communication can follow different philosophies. They can differ between a direct communication between the aircraft or an indirect which uses the GCS as a hub. Another design decision is whether to make the necessary software modifications directly in the existing autopilot control software or implement independent applications which run on a dedicated companion computer to prevent any modifications to the open source software. Each structure has its advantages and disadvantages.

A communication through the GCS requires a continuous data link between the aircraft and the GCS. Based on the final maneuver a beyond visual line of sight (BVLOS) flight may be required which could be a problem. The probability of losing a visual line of sight (VLOS) data link increases with increasing distance between the GCS and the aircraft. In opposite to this situation, the distance between the aircraft in the formation is nearly constant and independent from the distance to the GCS.

One approach for an air-to-air communication scheme is to use onboard companion computers. This would not require software modifications to the open source autopilot, but does have the disadvantage of added hardware and hence reduced payload.

Another possibility for an air-to-air communication scheme is to modify the open source autopilot. This option has the greatest advantages of direct air-to-air communication without additional hardware requirements. The disadvantage of requiring autopilot modifications is minimized through the use of an independent software library. Furthermore, the software library is only added on the master system. The slave system is free from any modifications.

The decision to use an air-to-air communication scheme leads to an extension of the avionic system of the demonstrators. An overview of the communication structure of the GNSS formation setup is shown in Figure 7. In addition to the communication between the GCS and the aircraft (green, dasheddot), a $433 \mathrm{MHz}$ link was added for air-to-air communication (blue, dashed). To prevent interference between the data links and avoid any bandwidth limitations, independent transceivers with different frequencies were used.

Both data links use the MAVlink protocol which is natively supported by the ArduPilot framework. Similar to the modifications of the autopilot, the modifications of existing MAVlink libraries (message structure or functions) should be limited as much as possible. Therefore, it was chosen to use existing standard MAVlink messages to implement the GNSS-based formation.

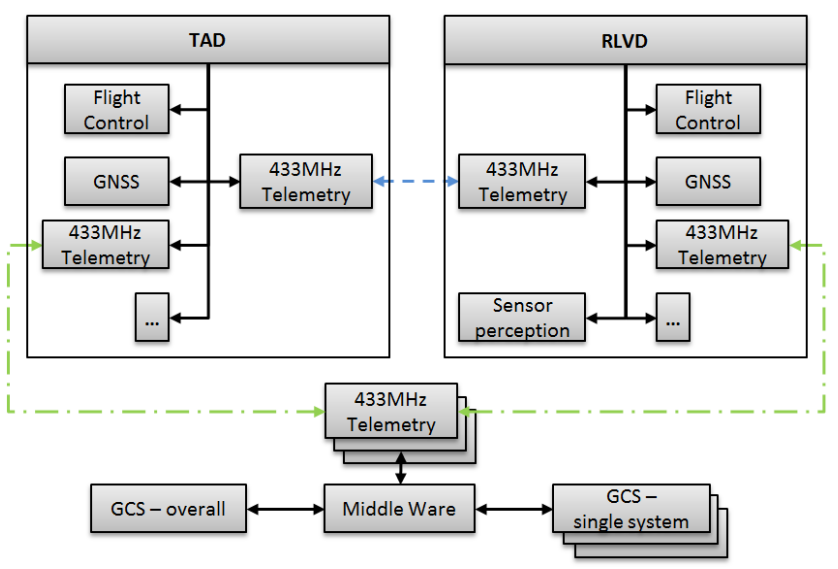

Figure 7: Communication set-up

The aim of the new software library is to set a basis which contains all the functionality necessary to build up the IAC maneuver in the presented setup. The first step of the overall IAC maneuver is to enable the GNSS-based formation between RLVD and TAD. The RLVD is the master system and the TAD represents the slave node. As described above the library runs as part of the RLVD autopilot and commands the TAD autopilot. 


\section{GNSS Formation Build Up}

The idea behind the GNSS formation is really simple. The RLVD does not have its own propulsion and cannot move directly to the location of the TAD. Therefore the TAD must move to the RLVD location and position relative to it.

This task contains two sub elements. The first is to set the TAD in a mode that enables it to follow external position commands. The second is to send commands and, if necessary, further information to enable a smooth, safe and reliable formation flight.

The first step, which is initialized by a GCS operator or pilot in command, is a request from the RLVD to the TAD to change the current flight mode to a guided flight mode. The guided mode is a standard mode of the ArduPlane application where the aircraft deviates from its waypoint mission to a specified point of interest.

The autopilot of the TAD provides a continuous heartbeat message that also contains its current mode. This heartbeat message is observed by the RLVD and used to confirm that the TAD has accepted the mode change. If the mode change was successful, the target position command is sent along with additional information.

The guided mode has the goal to fly to a geographic position of interest. Therefore, the target positions are specified in WGS84 coordinates. A target position is based on the current position of the RLVD including the altitude.

Providing the basic position of the RLVD makes less sense, because it will directly lead to a collision between the RLVD and the TAD. Therefore the RLVD position is modified with offsets to map different phases of the formation (approach, overtaking, flying ahead of the RLVD, etc.). The result is that the TAD can be positioned at each required position relative to the RLVD. The position command is updated at $20 \mathrm{~Hz}$.

Next to the position the RLVD provides a target velocity to enable a dynamic approach depending on the distance to the target location and to prevent overshooting. The TAD target velocity is based on the current velocity of the RLVD and a dynamic offset, which is modified by the distance between the current position and the target position of the TAD. The TAD provides its position by a standard $433 \mathrm{MHz}$ link which can be received by the GCS and the RLVD. The target velocity command is provided at a rate of $4 \mathrm{~Hz}$.

\section{Image Processing}

As described above, the IAC maneuver consists of two stages. The first stage is a rough formation approach using a GNSS-based positioning of the TAD led by the RLVD. The second stage has the aim to reduce the remaining position deviation between the ACD and the RLVD by using image sensor data.

\section{Overview and Hardware}

A limitation of the GNSS-based formation is the achievable accuracy: GPS accuracy with standard positioning services is
$7.8 \mathrm{~m}$ at $2 \sigma$. A differential GNSS solution between the RLVD and the ACD can deliver a more accurate positioning solution, with the same absolute accuracy. However, independent of the accuracy of the GNSS, it is not a good approach to rely on a single sensor. Rather, the solution should take into account additional independent sensors to detect the ACD to get several parallel comparable results.

In this project the ACD will be detected by an image based sensor system which consists of a monochrome camera and a Light Detection and Ranging (LiDAR) system. Both sensors are mounted in the nose of the RLVD, with a front view. They are oriented in such a way that they have an overlapping field of view (FOV) around the body-fixed x-axis of the RLVD. The mounting in the RLVD is an adaption that is necessary based on the size of the aircraft, since the ACD is too small to contain further sensors. In a real implementation of the IAC maneuver the sensor would likely be located in the ACD. This would help prevent a reduction of the RLV payload capacity and prevent a necessary opening for the sensors in the heat shield of the RLV. After current investigation, it is assumed that the different positions (client or ACD) of the image acquisition and processing have no influences to the entire IAC procedure.

The train of thought that an exclusive usage of GNSS at the identification of the ACD position wouldn't be a good approach was also the basis to use two opposite image generating sensor principles for the optical-based formation. Therefore a monocular grayscale camera and a scanning LiDAR were chosen which have complementary abilities to build up a comprehensive perception of the identical situation.

A grayscale camera is a cheap sensor which can achieve a high image resolution in a common configuration. This feature helps to easily identify the ACD in front of a dynamic background. This also leads to an accurate position estimation parallel to the camera plane in contrast to sensors with a lower resolution FOV. The position estimation of the ACD orthogonal to the camera plane (along the ray path) is not the best in general since the distances cannot be directly measured. The distance estimation along the ray path is only an approximation based on the intersection theorem under usage of the measured ACD size at the image plane and the known absolute ACD size.

In opposite to these abilities, the LiDAR can detect distances along the ray path quite well with direct, absolute measurements. In addition, the LiDAR emits light beams in the infrared spectrum which enables perception when visibility would otherwise be poor for the visual spectrum of the camera. Disadvantages of current scanning LiDAR systems are that they in general have a low image resolution and a low update rate as well.

The image processing has the goal to detect, identify and track the ACD using the respective image data. The following section describes an approximation of the ACD position relative to the RLVD. The processing of these steps is performed on the companion computer on the RLVD. The resulting target position is finally transmitted to the ACD. 
Based on this information, the ACD controller moves the ACD from its current position to the target position.

\section{Image Data Processing}

The processing occurs in parallel lanes each containing serial sub steps with the aim to handle different environment/ luminescent situations. The respective steps are combined with probability validation elements to obtain a relative position estimation of the ACD.

An overview of the image processing concept is shown in Figure 8 .

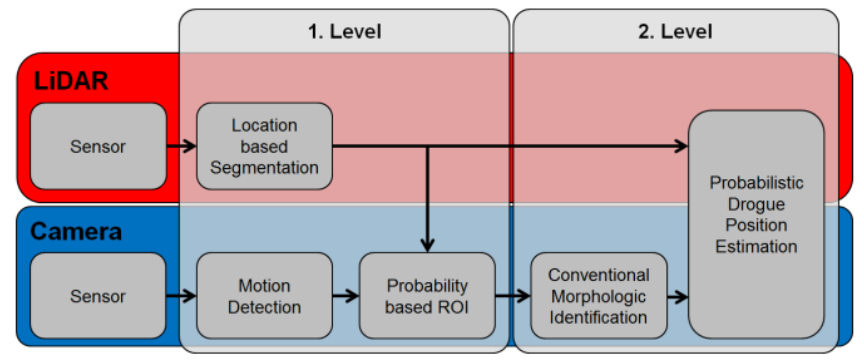

Figure 8: Image processing concept

The image processing is separated by the sensor signal data paths. One path covers the processing of LiDAR data and the other path contains the computation of grayscale camera data.

Motion Detection - The processing of the camera images starts with a motion detection approach. This will search for elements in the image, which are moving independently of the camera motion [11].

The implementation of this step is done by a relative motion detection of the image background between two successive images. The relative motion of the background is identified via the motion of features in the image background relative to the previous image. The features are equally distributed to be sure it is estimating the motion of the background. It is assumed that the background motion correlates with the camera motion. If objects in the image follow other/independent motions, these should differ from the background motion.

Based on the identified background movement, a coordinate transformation of the predecessor image is performed and the resulted image is subtracted subsequently from the current image. The resulting difference image shows objects which have moved independently relative to the background motion, and is likely the TAD or the ACD, and therefore the interest for further investigation.

LiDAR Data Segmentation - Parallel to the identification of interest regions in the camera image, the LiDAR data are segmented. An assumption of the LiDAR data processing is that the LiDAR field of view ${ }^{1}$ has a maximum sense range and in the case of the GNSS formation, a LiDAR beam can interact only with two objects, namely the ACD and the TAD. Additionally it is assumed, that based on their positions in the

1 The used LiDAR is a scanning system, which has a sensing field of $30^{\circ} \times 360^{\circ} \times 150 \mathrm{~m}$ and a resolution of $16 \times 2000$ measurements with a depth 7 formation, the ACD must be closer to the LiDAR sensor than the TAD.

Based on these assumptions, image segmentation was implemented to determine maximum two segments in the LiDAR data. The segmentation is based on the distance values of each measurement and respective of the neighborhood in the structured image grid. The assignment to the segments occurs with a maximum deviation threshold, which is limited to half the length of the towing rope between TAD and ACD. The result of the LiDAR data processing is two segments that are the initial point for further investigation.

The independent results of the camera and LiDAR perception are assessed and combined to a common result. The fused result is several regions of interest (ROIs) in the camera image plane that are fundamental for subsequent processing steps.

Morphologic Identification - Up to this point the described steps have the aim to identify the ACD indirectly through its motion or location relative to the TAD. A further approach is the morphologic identification by its shape or design. This approach was implemented in the second level of the image processing.

For the conventional morphologic identification it is required that the GNSS-based formation was successfully implemented and the camera image shows the ACD from behind. As shown in Figure 5, the rear part of the ACD is a cone and a planar projection of the cone base results in a circle. Therefore, it is assumed that if the ACD is observed from the RLVD position in the formation, that the projection of the ACD at image plane has approximately the shape of a circle. Finally, this circle is used to identify the ACD as well. The circle estimation uses a Hough [12] approach to identify pixels which form a part of different circles. The respective circles differ in the location of their center and their radius. The circle estimation does not run across the whole camera image, rather only in the ROIs identified in the previous processing steps.

ACD Position Estimation - In the final step of the image processing the results of the different steps are assessed collectively. The results of the individual steps are available in camera image coordinates and contain additional values, such as the respective LiDAR distance and the estimated probability that the described object is the ACD. Based on coordinates and further values, spherical object coordinates are used to determine the coordinates of the probable ACD in the RLVD coordinate frame.

\section{EXPERIMENTAL ANALYSIS}

The presented steps to fulfill the IAC approach, development of the ACD, the GNSS-based formation and the image based ACD detection, were evaluated in separate tests. The evaluation of the ACD development will be shown with flight test at which an exemplary aircraft tows the ACD. The

resolution of one centimeter. 
implementation of the GNSS-based formation will be evaluated with a flight test of two small unmanned aircraft flying a race track pattern. The results of the image processing will be presented with ground and flight test, which are implemented with different aircraft systems, such as multi-copters and airplanes.

\section{ACD Motion Analysis}

The ACD was evaluated with regard to its ability to be towed behind a small unmanned vehicle, its flight behavior, and its controllability. One part of the control law of the ACD contains a roll stabilization, which will be assumed to be working properly and will not be part of the following evaluation.

The objective of the following section was to achieve as much horizontal and vertical movements of the ACD as possible when deflecting the control surfaces.

Flight Test Setup — The flight test was implemented with different setups. Depending on the requirements of each test, a different flight test demonstrator was chosen.

An exemplary setup, which was used for the ACD displacement evaluation, was a towing aircraft configured with a onecylinder combustion engine and an autopilot to automatically fly waypoint missions in addition to its remote control connection. The flights to validate the ACD were performed at speeds of about $140 \mathrm{~km} / \mathrm{h}$ and with a rope length of $30 \mathrm{~m}$. Figure 9 shows the towing aircraft, and Table 2 presents its technical data.

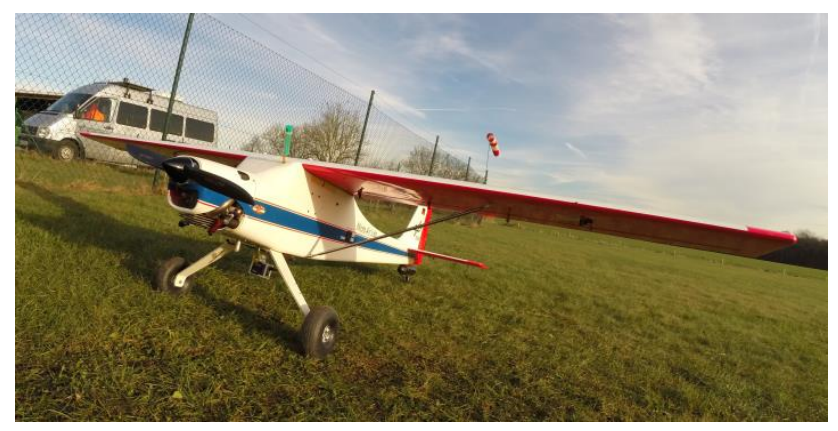

Figure 9: Towing aircraft $\mathrm{MAL}^{2}$

Table 2: Technical data of the towing aircraft MAL

\begin{tabular}{|l|l|l|l|}
\hline Wing span & $2.7 \mathrm{~m}$ & Max. altitude & $600 \mathrm{~m}$ \\
\hline MTOW & $17 \mathrm{~kg}$ & Displacement & $62 \mathrm{~cm}^{3}$ \\
\hline $\mathrm{V}_{\max }$ & $160 \mathrm{~km} / \mathrm{h}$ & Engine power & $3.67 \mathrm{~kW}$ \\
\hline $\begin{array}{l}\text { Max. flight } \\
\text { time }\end{array}$ & $30 \mathrm{~min}$ & & \\
\hline
\end{tabular}

During the flight test, the towing aircraft continuously flew a racetrack pattern, as shown in Figure 10. The racetracks were performed in altitudes between 70 and $100 \mathrm{~m}$ above ground level. The leg length is approximately $500 \mathrm{~m}$, and the distance between the legs is $150 \mathrm{~m}$. The available time for a straight leg of the course without wind influence is between 13 and $15 \mathrm{~s}$.

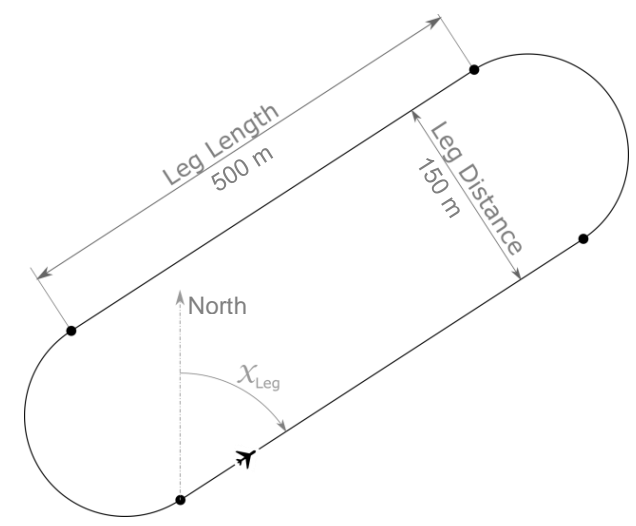

\section{Figure 10: Racetrack mission for performing the IAC} maneuver

The flight tests for evaluating the ACD flight behavior and the controllability were performed on the described racetrack.

All measured data were recorded separately by the autopilot of the towing aircraft and the ACD. The sensors used include a GNSS/compass and the internal inertial measurement unit of the Pixhawk devices.

Vertical Displacement - The determination of the achievable vertical movement was performed during the flight tests. An example of the logging data from these tests is shown in Figure 11 .

The upper graph of Figure 11 compares the towing aircraft altitude $\left(\mathrm{h}_{\mathrm{MAL}}\right)$ and the altitude of the $\operatorname{ACD}\left(\mathrm{h}_{\mathrm{ACD}}\right)$. It can be observed that the ACD follows the MAL with a deviation in altitude of around $\pm 3 \mathrm{~m}$ until the time $\mathrm{t}=510 \mathrm{~s}$, when the controller is activated. This is also observed in the middle graph, which shows the altitude deviation between MAL and $\operatorname{ACD}\left(\mathrm{h}_{\mathrm{MAL}}-\mathrm{h}_{\mathrm{ACD}}\right)$. Beginning at $\mathrm{t}=510 \mathrm{~s}$ the horizontally oriented control surfaces are manually deflected upwards and downwards, shown as remote control vertical input (RVIN) in the bottom graph of Figure 11. RVIN inputs generate indirect altitude change. 

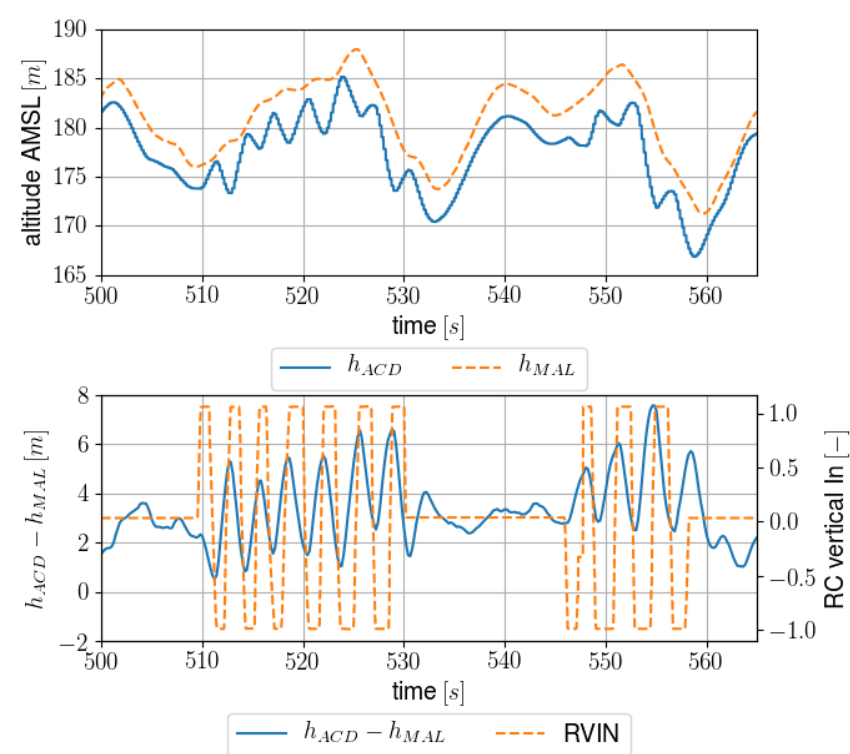

Figure 11: Altitude and altitude offset during vertical motion

The inputs result in a vertical displacement of the ACD with respect to the towing aircraft, visible as oscillations in the absolute altitude and in the relative altitude deviation between both vehicles. The deflections from RC inputs are limited to $90 \%$ of the possible deflection of the controls to maintain sufficient control authority for the roll stabilization. Figure 11 shows a typical displacement of $+/-3 \mathrm{~m}$. Due to the strong winds and turbulence effects during the tests it was not possible to evaluate the data for longer control inputs as wind displacements strongly interfere with the controlled displacements.

Horizontal Displacement - Displacements in horizontal direction were performed in the flight test similarly to the vertical movements. As described above, the experiments were performed while flying an automatic racetrack mission, shown in Figure 12.

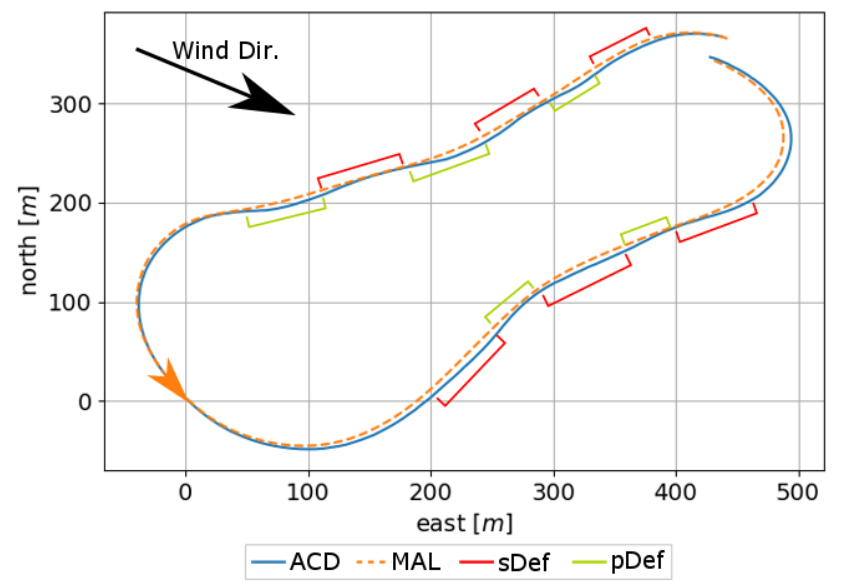

Figure 12: Flight path during horizontal movements

During the experiments, the wind speed was very high with speeds between 20 and $28 \mathrm{~km} / \mathrm{h}$ from the west-northwest direction, shown in Figure 12. During the mission, manual inputs to move the ACD in the horizontal direction were carried out while the roll stabilization was active. The control inputs are shown as a red line (sDef) in Figure 12 for the starboard inputs and as a green line (pDef) for the port inputs. Because it is difficult to make quantifiable statements from Figure 12, Figure 13 shows additional information for the same time range.

Figure 13 shows the RC inputs and the relative horizontal displacement of the ACD with respect to the momentary track of the towing aircraft. The dependence of the position on the inputs can clearly be seen. Due to the strong northwesterly wind, the relative position of the ACD to the towing aircraft track is constantly shifted to one side even without a control surface deflection. This can be seen in the graph where the displacement is constantly positive on the first leg and constantly negative on the second leg of the racetrack. During the first part of the racetrack, the shift is towards the left side of the towing aircraft track, while after the turn, on the second leg, the shift is towards the right side.

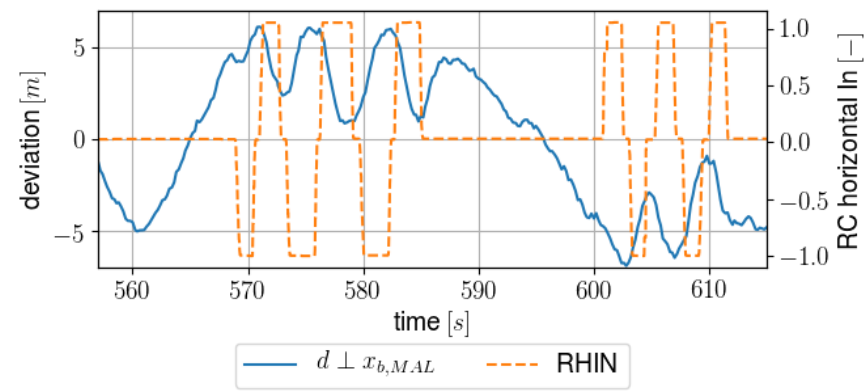

Figure 13: Horizontal displacement during the horizontal movements

The displacement by constant wind is the reason why the movements are not equally distributed around zero in the graph of Figure 13 but have an offset. The displacements due to control inputs from this offset can be estimated to be up to $2.5 \mathrm{~m}$. Given the size of the coupling unit and the towing aircraft and also the wind conditions, this result for possible displacements is very satisfying and agrees well with the data from the vertical displacement tests.

The main result of the flight tests for the vertical and horizontal movement is that the respective displacement with respect to the towing aircraft position is within $+/-2.5-3 \mathrm{~m}$ in the expected range and allows compensation of position offsets to the client. This becomes clear when the wingspan $(2.7 \mathrm{~m})$ of the towing aircraft in relation to the displacement is considered. The attitude of the coupling unit is not constant during the movements. As the cone of the configuration compensates the changes in pitch, problems for the capturing maneuver are not expected.

\section{GNSS Formation}

The objective of the following section is to validate the ability to initiate and control a GNSS-based formation between two aircraft. Specifically, it should investigate how the aircraft can follow predefined parameters under external influences. 
Flight Test Setup - The evaluation of the GNSS-based formation implementation uses small unmanned, electric powered aircraft of type "Volantex RangerEx 757-3" to show the procedure of the formation approach. Each plane was equipped with Pixhawk 2.1 cube, u-blox M8N GNSS module, Holybro $433 \mathrm{MHz}$ telemetry and an airspeed sensor.

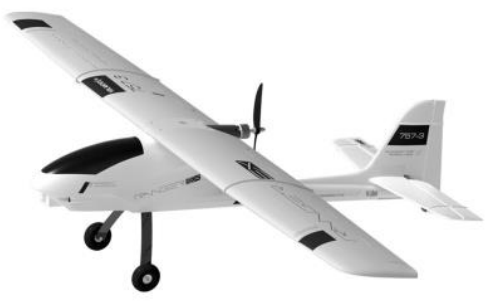

Figure 14: Volantex RangerEx 757-3

The initial situation to validate the implementation of the GNSS formation is that two UAVs are in air and follow two different automated initial maneuvers. The master UAV follows a waypoint mission which corresponds to the racetrack pattern described above. The slave aircraft loiters in a circle outside the racetrack, waiting for further instructions. An overview about this mission setup is presented in Figure 15. The orange race track describes the path of the master and the red circle with approach path shows the behavior of the slave. The gray areas show the location of the runway (rectangle) and the crew (circle).

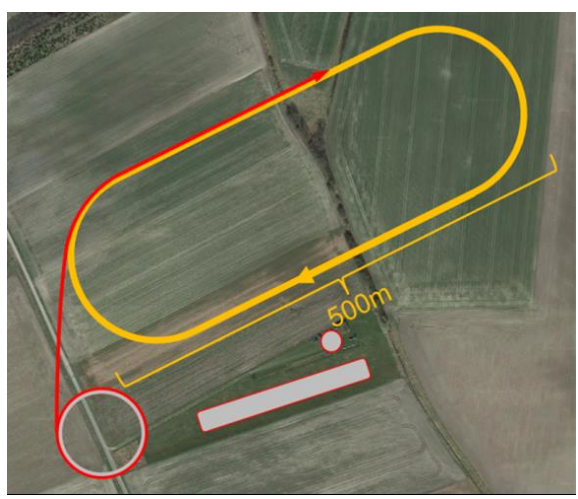

\section{Figure 15: Overview of the GNSS formation mission setup}

Formation Procedure - Initiated by the pilot in command (via RC or GCS) of the master, the modified master autopilot transmits a request to the slave, with the aim to change its flight mode to guided mode. When the slave receives this request, it will follow the command and will accept subsequent target positions and velocity commands.

In the current case, the slave receives the target position, which is located $40 \mathrm{~m}$ behind the master along its heading direction.

The target velocity is based on the distance between slave and master and varies between the maximum velocity of the slave and the current velocity of the master. The distance at which the predetermined differential speed becomes zero, combined with the proportional gain $K_{P}$, which describes the ratio of commanded airspeed difference to relative distance between 10 the two UAVs, is the most sensitive parameter. A gain of $K_{P}$ $=0.027$ to 0.03 has proved successful. This means that at a distance of $100 \mathrm{~m}$, a commanded differential speed can be 2.8 $\mathrm{m} / \mathrm{s}$ up to $3.0 \mathrm{~m} / \mathrm{s}$. The maximum speed offset for the slave is limited to $7 \mathrm{~m} / \mathrm{s}$, which is hardly a restriction. Due to delays in the radio link and the control, there is additional deviation leading to the slave lagging behind the target point. The dynamics are strongly influenced by battery level and wind susceptibility of the aircraft. With the above values, the best results were achieved.

Figure 16 shows the relative $\mathrm{x}$-distance between the master and slave in the body fixed coordinate system of the master over a complete flight. It was recorded by the master system based on its own position estimate and the position transmitted by the slave via telemetry. The area was marked in which the formation flight was active. The formation was active in this flight over a period of $130 \mathrm{~s}$ (several flights were carried out, one of which is evaluated here as an example).

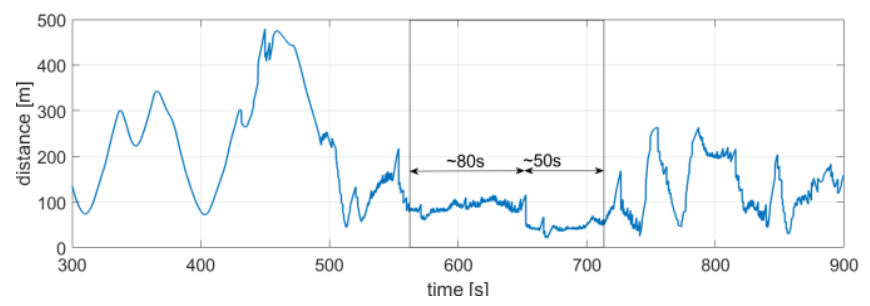

Figure 16: $x$-Deviation between Slave and Master

The time range in which the formation was active is shown enlarged again in Figure 17. In addition to the x-distance between both participants, the roll angle of the master aircraft is also shown. The logged formation, shown in Figure 16, can be divided into two phases. At the first phase, the slave follows the master with a distance of approx. $80 \mathrm{~m}$ over a period of 80 seconds. In the second phase, the distance decreases to $40 \mathrm{~m}$. This phase has duration of $50 \mathrm{~s}$. The difference between both phases is a modified speed command, which is caused by an increase of the gain $K_{P}=0.027$ to 0.03 at the beginning of the second phase.

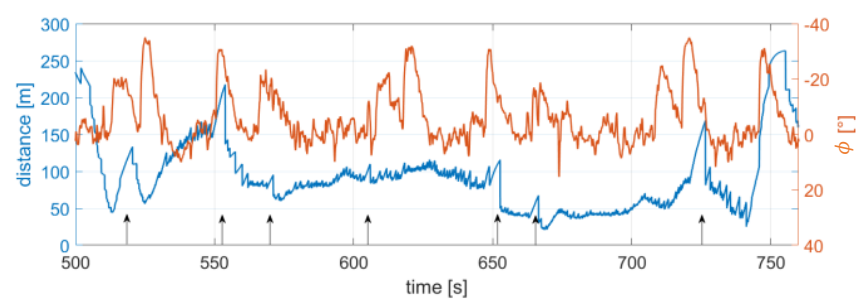

Figure 17: x-Deviation between formation participants and roll angle of master

Overall a good formation can be achieved between both aircraft in both periods. The respective distance varies between $5 \mathrm{~m}$ and $10 \mathrm{~m}$ in straight flight and slightly increases in the turns, which is due to the nature of the command. 
One striking feature of the time courses are the sudden position changes (marked by the arrows). It can be observed that the distance between master and slave increases continuously (sometimes up to very high values, e.g. at $550 \mathrm{~s}$ ) and suddenly drops back to "normal" level. The reason for these peaks is the performance of the telemetry link. The increase of the deviation is due the lack of updated data. The master who calculates this distance knows its current position but doesn't receive an updated slave position. This in turn increases the calculated distance, as it was obtained with an outdated slave position. When new data are received the calculation suddenly jumps to the actual value. The result of the chosen implementation is that each connection loss leads to an increased velocity suggestion. If this command arrives at the slave, or is transmitted directly when the connection is re-established, the slave reduces the distance until the actual distance is transferred and leads to a new speed suggestion. This distance decrease can be observed after each connection abort. This is especially visible in the stable phases, e.g. at $570 \mathrm{~s}$ and $670 \mathrm{~s}$.

To determine the cause of the connection loss, Figure 17 shows also the roll angle of the master aircraft. It becomes clear that peaks in the distance plot, caused by missing data updates, correlate with peaks of the roll angle plot. This observation can't explain all distance peaks, but a connection is clear. The antenna of the telemetry, used for the formation, is attached to the right side of the fuselage. One possible cause of the shown relationship is that the fuselage shields the radio link in curve flights what leads to a bad connection up to a loss.

\section{Image Processing}

The image based estimation of the ACD position at the final approach of the IAC maneuver was validated with ground and flight test. The objective of the following section is, to validate the identification of the ACD in the data and the interaction of the LiDAR and camera perception.

Test Setup - The test setup is separated into ground and flight test. The ground tests have the goal to generate easily perceivable data to enable an early development of the algorithms, to show the interaction of the LiDAR and camera perception and to validate the results of the individual algorithms. The flight tests used a simplified implementation which provides only a camera perception, but gives an insight into a more realistic implementation of the final scenario.

For the ground test, a portable setup was built which contains sensors (camera and LiDAR), processing unit and power supply, which are identical with the setup in a planned RLVD. The portability enables a positioning at different raised locations to observe flying objects. In the here described implementation, the flying object is a multi-copter, which was equipped with a simplified ACD dummy. The dummy consists only of the trailing cone and enables a dedicated positioning of the cone in front of the sensors.

The used grayscale camera has a resolution $1024 \times 800$ and a pixel size of $2.5 \mu \mathrm{m} \times 2.5 \mu \mathrm{m}$. The lens has a focal length of 11
$4.9 \mathrm{~mm}$. A Velodyne Puck LITE, with a $30^{\circ} \mathrm{x} 360^{\circ}$ field of view, 16 px x 2000 px field resolution and a max. measurement range of $120 \mathrm{~m}$ with a resolution of $0.01 \mathrm{~m}$, was used as LiDAR sensor. Figure 18 shows a CAD sketch of the used sensor setup, which is also used at the RLVD.

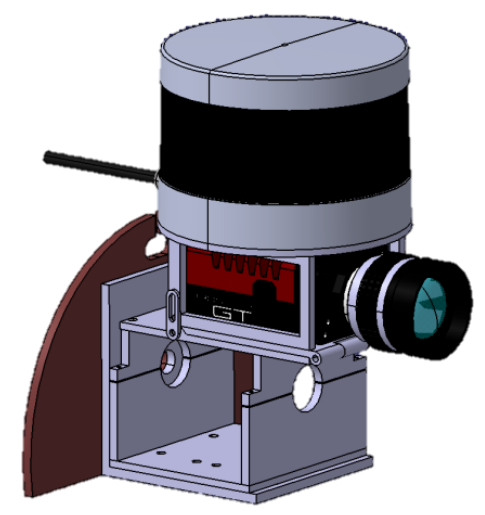

\section{Figure 18: Mounting of LiDAR and camera in the RLVD}

An exemplary camera image of the recorded situation, including the multi-copter equipped with the ACD dummy is shown in Figure 19. The marked area in the center of the image represents the projected field of view of the LiDAR.

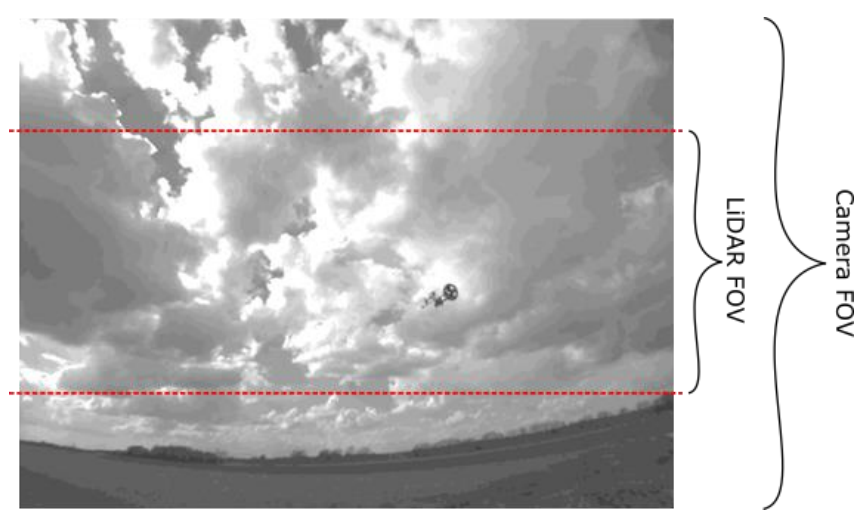

Figure 19: Example situation at the ground test for the image processing

The flight test setup is based roughly on the setup which was presented at the validation of the GNSS formation. The same planes with the same avionics were used. A difference is that the setup was extended with an ACD dummy. The dummy used differs from the one used during ground tests. It is considerably lighter and smaller, because the small electric powered aircraft are not powerful enough to tow the developed ACD. Therefore, a large shuttlecock with a cone base diameter of $0.15 \mathrm{~m}$ without avionics and control surfaces was used. An exemplary image of the observed situation with the ACD and the towing aircraft is shown in Figure 20. 


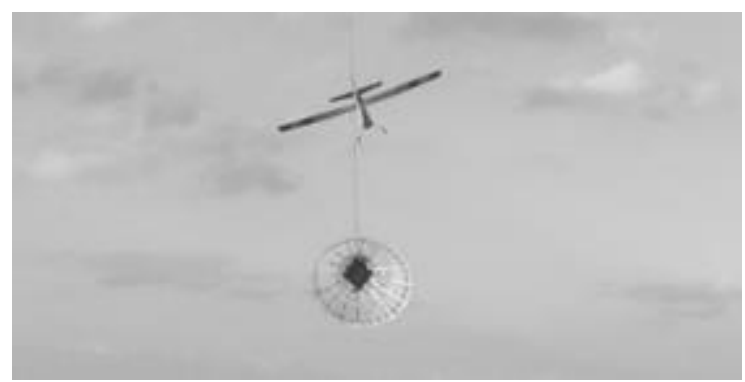

Figure 20: ACD flight test setup for image data acquisition

In addition, it was not possible to equip the small electric powered aircraft with the original sensor hardware which is planned for the final RLVD demonstrator. The camera was substituted with a GoPro 5 camera with a resolution 1280px by $960 \mathrm{px}$, which is comparable to the original camera. The LiDAR system is too heavy, so the integration was not possible. The small aircraft size also prevented the integration of further computing hardware, with the result, that the data were processed offline after the flight tests.

Ground Test Procedure and Result - As described above the goal of the ground tests was to evaluate the combination of camera and LiDAR processing. Specifically the ability to detect the same object in a common field of view with different perception technologies to enable a data fusion at complementary and competing information states should be demonstrated.

The detection of an object, the ACD, with different technologies and subsequent fusion of respective data in a common coordination frame requires that the data/ information be transformed into this common system. In the here described case, this system is the image plane of the camera. The transformation is based on the knowledge about the intrinsic and extrinsic parameters of each sensor and between both. These parameters will be assumed as known and their determination is not part of this paper.

The sensor data recorded during the ground tests were postprocessed offline after the tests.

For the camera processing, the described motion processing with a subsequent morphologic identification on the basis of Hough circle detection was used to finally obtain spherical coordinates of the ACD. For the LiDAR data the computation uses the segmentation approach followed by the transformation of the results into the camera frame and finally into spherical world coordinates.

These procedures were applied on six data sets with different background situations (variants of clear sky, cloudy sky and sunlight). For all these sets the detection of the ACD was successful in $85 \%$ of the images if the ACD was visible in the overlapping field of view.

Correct detection means in this case that the candidate in the current image can be combined with a candidate from previous images by the tracking algorithm and the resulting / updated candidate with the highest traceability value is the ACD. The here presented approach assumes that each track finds the ACD and other false candidates. But the tracking and the subsequently comparative fusion identify the wrong candidates and ultimately only the true ACD remains as the only solution.

A closer look to the results of camera and LiDAR processing is shown in Figure 21. The results are based on a data set which was chosen exemplary for all recorded ground test data. The figure contains the resulting spherical world coordinates of camera and LiDAR processing in the camera coordinate frame. In addition to the visual sensor data, the GNSS data are plotted for $\phi$ and radius values. The GNSS data are based on the recorded flight logs of the multi-copter and provides a rough estimate whether the data from the optical perception are in the right range. The multi-copter controller estimates the altitude via GNSS and barometer. These estimations are very bad in general which is why the $\theta$-chart does not have a GNSS plot.

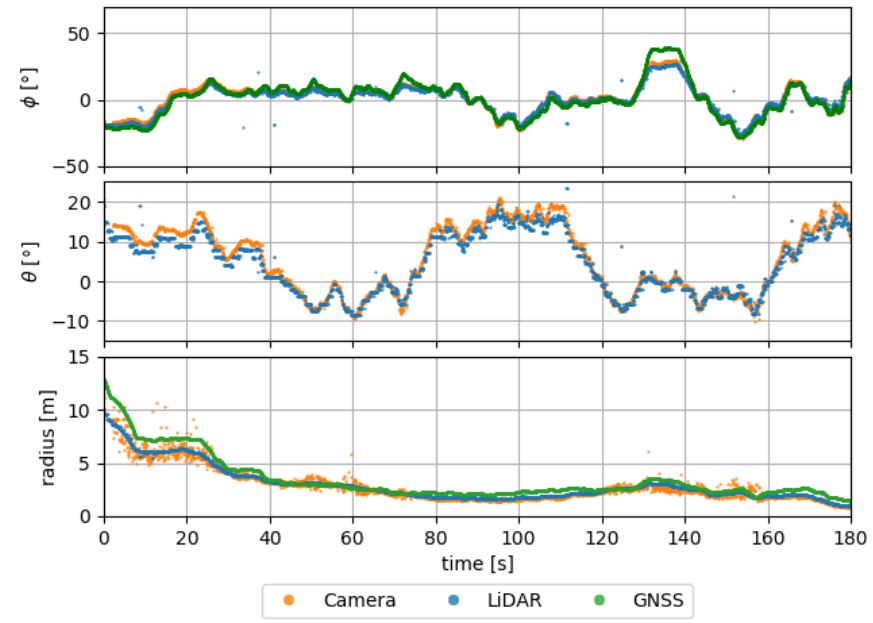

\section{Figure 21: Optical position estimation of $\mathrm{ACD}$ in rela- tion to GNSS}

Generally, the three charts of Figure 21 show a good correspondence between the optical sensor sources, also in relation to the GNSS. A more precise view is shown in Figure 22, which contains the computed delta between the camera and LiDAR measurements as well as the LiDAR and GNSS measurements. 

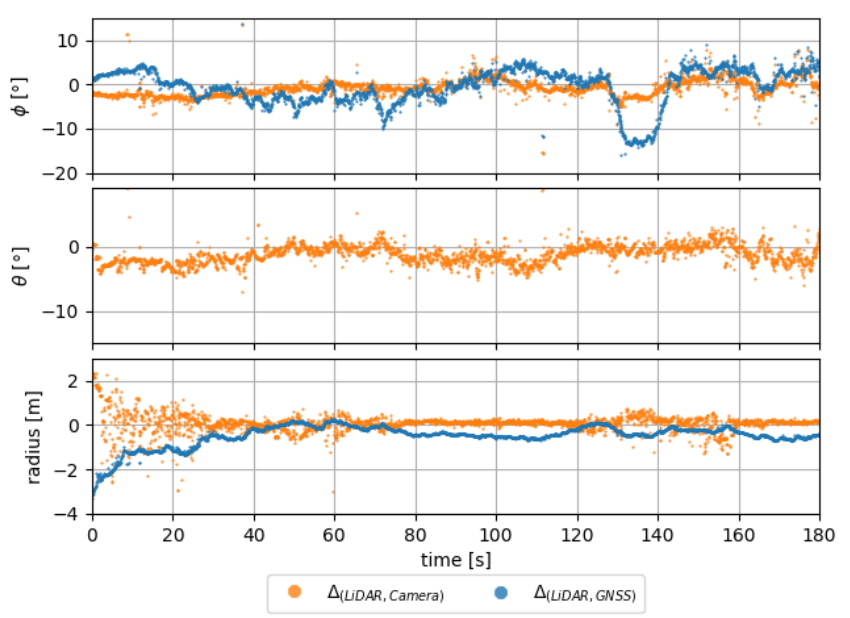

Figure 22: Delta between the optical position estimations and GNSS measurements

The $\phi$ and $\theta$ axes show a good correspondence between the optical sensor data and the delta in general, with the exception of some outliers, remains less than 5 degrees. The radius delta between the LiDAR and camera does not look clean and low. It can be seen that, especially for longer distances ( $t=0$ up to $\mathrm{t}=35 \mathrm{~s}$ ), the delta increases and is noisier, compared with Figure 21. This behavior was expected and is based on the indirect distance estimation on the camera data under usage of the intercept theorem and the increasing influence of the pixel size with increasing distance. For shorter distances ( $t=75$ up to $t=120 \mathrm{~s}$ ) this problem has less influence. The knowledge about this behavior was the basis of the decision to use the absolute measurements of the LiDAR additionally to the camera.

The cause of deviations between the LiDAR and the GNSS data cannot be clarified conclusively, but they are less than a few meters $(<3 \mathrm{~m})$, which is within the accuracy of a standard GPS solution. That is why it was assumed that the delta is based on the noise of the GNSS measurements and not on the LiDAR measurements.

The ground tests present, in general, good results for the optical sensor perception. But they are ground tests and there are some differences to a flight scenario. One is the fixed position and state of the camera. During a flight of an aircraft its position changes continuously and therefore the position of the camera as well. The consequence is that algorithms such as the motion perception, the first step in the camera data processing lane, runs during the ground test at a simplified situation. The difference is that during the ground tests the motion perception should not detect any motion of the camera or background, except minimum movements of the clouds. This situation is completely different during a flight test; the image background is continuously changing and more challenging for the algorithm.

Flight Test Procedure and Results - The conducted flight tests are described in the sections above. They were completed with three tests using the same setup. The recorded 13 data was processed offline with the described camera algorithm lane. Ground truth data does not exist; this is why at the current section only the identification of the ACD is taken into account.

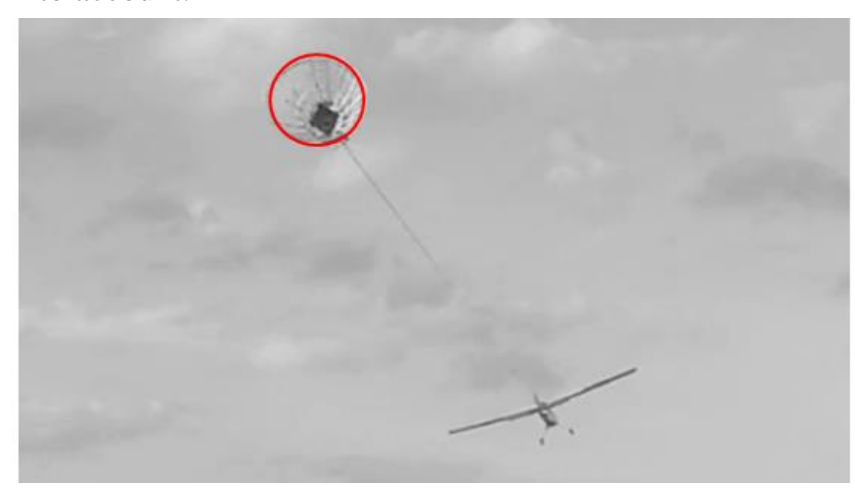

Figure 23: Example Image of a flight test situation

The validation of the data showed a slightly lower detection rate of the ACD in the flight test data in comparison to the ground test. A detection rate of $77 \%$ could be achieved.

\section{SUMMARY \& OUTLOOK}

The presented work gives an overview about early results of investigations which explore the feasibility of an in-air capturing approach of a reusable launch vehicle by a separate aircraft. Because the investigations are in a very early state, initial studies were implemented with a scaled setup. Starting from this scaled setup, three subtasks were presented: the development of an automatic controlled coupling devise, the necessary formation flight between the two aircraft and an optical perception of the automatic coupling device for the last stage of the coupling approach. All three tasks were presented with concepts, the implementation and some of the ideas behind each. In addition, each task was validated with flight or ground test to show the expected functionality and whether the requirements behind the concepts can be fulfilled. The presented work gives a first overview about the investigation and shows the work in progress.

The next steps are to tune the mission control of the GNSSbased formation flight to generate a more stable formation. This step is also a requirement to get further and better perception data for the second phase of the approach and compare the optical perception results with the flight logs of the aircraft. The final goal is to bring the perception processing into the aircraft and implement the whole IAC coupling approach in one mission.

\section{ACKNOWLEDGEMENTS}

Part of this work was performed within the project 'Formation flight for in-Air Launcher 1st stage Capturing demonstration' (FALCon) addressing development and testing of the "in-air-capturing" technology. FALCon, coordinated by DLR, is supported by the EU within the Horizon2020 Program 5.III. Leadership in Enabling and Industrial Technologies - Space with EC grant 821953. Further information on FALCon can be found at http://www.FALCon- 
We thank all of our colleagues who supported us during the investigations and the ground and flight test campaign. Special thanks go to our safety pilots Michael Kislat-Schmidt, Jörg Rößner and Pascal Lipke. In addition, we want to also thank the master students Asija Velieva and Andreas Seel.

\section{REFERENCES}

[1] SpaceX, [Online]. Available: https://www.spacex.com/reusability-key-makinghuman-life-multi-planetary. [Accessed 0412 2019].

[2] L. Bussler and M. Sippel, "Comparison of Return Options for Reusable First Stages," in 21st AIAA International Space Planes and Hypersonics Technologies Conference, Xiamen, China, 2017.

[3] M. Sippel, C. Manfletti and H. Burkhardt, "LongTerm/Strategic Scenario for Reusable Booster Stages," in 54. International Astronautical Congress IAC, Bremen, 2003.

[4] M. Sippel and J. Klevanski, "Progresses in Simulating the Advanced In-Air-Capturing Method," in 5th International Conference on Launcher Technology, Cologne, 2003.

[5] S. I. Purdy, "Probe and Drogue Aerial Refueling Systems," in Encyclopedia of Aerospace Engineering, John Wiley \& Sons, Ltd, 2010.

[6] T. Kuk, K. Ro and J. Kamman, "Design, Test and Evaluation of an Actively Stabilized Drogue," in American Institute of Aeronautics and Astronautics 2011-1423, St. Louis, Missouri, 2011.

[7] D. Sims-Williams and R. Dominy, "Improved Air to Air Refuelling," in Trans National Access Opportunity Workshop, Bardonecchia, 2011.

[8] W. Williamson, E. Reed, G. Glenn, S. Stecko, J. Musgrave and J. Takac, "Controllable Drogue for Automated Aerial Refueling," Journal of Aircraft, pp. Vol. 47, No. 2, March 2010.

[9] P. Eldon, "Drogue for airspeed-calibration trailing static source". USA Patent US3310257, 1st October 1965.

[10] PixHawk, "Flight controller products," [Online]. Available: https://pixhawk.org/\#autopilots. [Accessed 0412 2019].

[11] C. Briese, A. Seel and F. Andert, "Vision-based detection of non-cooperative UAVs using frame differencing and temporal filter," in International Conference on Unmanned Aircraft Systems, Dallas TX, UAS, 2018.

[12] J. Illingworth and J. Kittler, "The Adaptive Hough Transform," IEEE Transactions on Pattern Analysis and Machine Intelligence, vol. 9, no. 5, pp. 690-698, 1987.

\section{BIOGRAPHY}

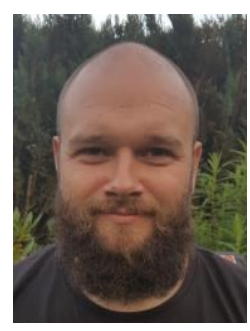

Stefan Krause studied economic computer science at the technical university of Braunschweig, Germany. He finished the studies with a Diploma (Master) degree in 2008. Subsequently he worked as $\mathrm{PhD}$ student and scientist at the German Aerospace Center (DLR), Institute of Flight Systems, department of unmanned Aircraft, until now. He graduated in 2016 with a $\mathrm{PhD}$ in the field of environment perception and noncooperative obstacle detection for unmanned aircraft. During the time at DLR his work comprises the participation in several projects in the field of environment perception with camera and LiDAR for unmanned aerial vehicles (UAV), lead of internal projects or major work packages, and the planning and implementation of flight tests of rotary and fixed-wing UAV as well as multi vehicle scenarios.

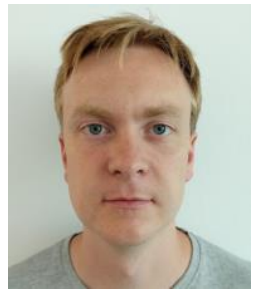

Sebastian Cain started as an aircraft mechanic in the long-range fleet at Lufthansa Technik and later obtained his bachelor's and master's degree in aerospace engineering at the technical university of Berlin. He was working at the technical university of Berlin in projects for formation flight control with two commercial aircraft and in the field of thrust control by nx-commands. Before starting at DLR he was working for Stemme AG as design engineer for motor glider aircraft and was involved in type and change certification as well as research for the certification process of an electronic flight control system for CS-23 aircraft. At DLR he works with unmanned aircraft in the fields of air-to-air refueling and formation flight and is involved in flight tests as well as simulation campaigns. 